\title{
Evaluation of Physical Education Teaching Quality in Colleges Based on the Hybrid Technology of Data Mining and Hidden Markov Model
}

\author{
https://doi.org/10.3991/ijet.v15i01.12533 \\ Yuansheng Zeng \\ Chongqing Technology and Business University, Chongqing, China \\ $895381769 @ q q . c o m$
}

\begin{abstract}
In order to further improve the teaching quality evaluation accuracy of physical education(abbreviated as PE) curriculum in colleges, this study conducts an in-deep research on the overall evaluation of PE teaching effect in colleges from the aspects of teachers' teaching ability and students' learning effect based on the hybrid technology of data mining and hidden Markov model. First of all, this study analyzes the development status of the teaching quality evaluation system of PE curriculum in colleges; Secondly, it analyzes the applicability of data mining technology and hidden Markov model to the evaluation of PE teaching quality in colleges, and proposes a mathematical model for evaluating the quality of PE teaching in colleges; Finally, this study carries out a series of experiments on the basis of mathematical models, and analyzes the experimental results in depth. The experimental analysis shows that the model proposed in this paper is helpful to improve the accuracy of PE teaching quality evaluation in colleges. The research results of this study provide a useful exploration for the integration of computing technology and language teaching. At the same time, it provides a reference path and implementation model for improving the teaching of PE for graduates in colleges through machine learning technology.
\end{abstract}

Keywords - Data mining, hidden Markov model, PE for graduate students, teaching evaluation.

\section{Introduction}

In order to further improve the teaching quality evaluation accuracy of PE curriculum in colleges, this study explores the overall evaluation of PE teaching effect in colleges in depth based on a detailed analysis of the applicability of machine learning technology to improve PE teaching and with references to mining technology and hidden Markov model.

There has been a long history of the research on the teaching evaluation. Among the available references, earlier research dates back to the middle of the last century [1-2]. Among the subsequent studies, some analyzed the evaluation of the special PE teaching effect in depth, some attempted to use practical methods to guide students to 
improve their self-evaluation ability, and some analyzed the influence of the composition and feedback mechanism of PE curriculum on the evaluation effect [3-8]. In addition, more recent research results also include the design and optimization of evaluation scales based on the actual needs of PE curriculum teaching [9], and how feedback can be formed through questionnaires to improve teaching [10].

Although a lot of research has been done on PE teaching evaluation by the predecessors, there is still room for further improvement in the accuracy and efficiency of teaching evaluation.

As to the research methods for PE teaching evaluation in colleges, the existing problems are as follows. Firstly, limited by cross-domain system thinking, traditional research methods in the field of social sciences are mainly adopted with little or no reference to the latest research results in the computing field, thus it is difficult to increase accuracy significantly. Secondly, the over-singular evaluation layer fails to reflect the indispensability of either the teachers or the students in the teaching process, and the education concept with students as the main body. In order to improve the performance of PE teaching evaluation algorithm, this article proposes a PE teaching evaluation model based on hybrid technology of data mining and hidden Markov model. Through the comparison with the previous methods, the advantages of the proposed algorithm in precision and computational efficiency are verified.

\section{Analysis on Applicability of Machine Learning Technology}

\subsection{Applicability analysis of data mining technology to the evaluation of PE teachers' teaching ability in colleges}

The data mining technology in the field of machine learning can effectively reduce the noise point interference of the data collected in the teaching process, and excavate more objective and useful information from the data base, thus improving the evaluation accuracy of teachers' teaching ability in teaching evaluation, and providing more reliable basis for subsequent teaching improvement.

Due to the differences in the professional level and personality of teachers, the teaching forms of PE courses in colleges are diversified. Among them, there are many noise points that affect the real evaluation of teachers' teaching ability, or even the opposite evaluation of teachers' teaching ability. Through manual methods, it is obviously difficult to effectively eliminate interference from noise points. Especially when the number of noise points is large, these methods are obviously not feasible for engineering applications. The data mining technology in the field of machine learning can effectively remove noise points in batches and extract valuable information from a large amount of data. Overall, the data mining process for evaluating teachers' ability to teach can be divided into the following five stages.

Stage 1: Data cleaning: This stage specifically includes preprocessing operations such as data selection, data discretization, and data normalization. The cleaned data set can be defined as: 


$$
D_{i}=\left[\begin{array}{llll}
d_{i 1} & , \cdots & , & d_{i j}
\end{array}\right]
$$

where, $D_{i}$ represents the $i$-th sample of the data set, $d_{i l}$ the 1 st attribute value of the sample vector $D_{i}$, and $d_{i j} 1 \mathrm{st}$ attribute value of the sample vector $D_{i}$, indicating that the dimension of the sample vector $D_{i}$ is $j$.

Stage 2: Soft Interval: In order to further improve data fault tolerance, a soft interval mechanism is introduced, which allows a small number of samples to not satisfy the constraint.

$$
w^{\mathrm{T}} D_{i}+b \geq \xi_{i}
$$

where, $w$ represents notation vector, $b$ the displacement variable, and $\xi_{i}$ the $i$-th slack variable.

Stage 3: Mining of data association rules: The mining of the association rules between the data means that the association rules between the conditional attribute set $A$ and the decision attribute set $B$ are solved in the sample vector set $\tilde{D}$ after the soft interval. First, randomly select a subset of conditional attributes, $A_{l}$; Then, calculate the association rules of $A_{l} \rightarrow B$; After that, iteration is continued until the change of the condition attribute subset $A_{k}$ of the $k$-th time is lower than the minimum confidence $\varepsilon$; Finally, use the current income association rules as mining results.

Stage 4: Kernel linear dimensionality reduction: In order to improve the computing speed and reduce the memory consumption, the kernel linear dimension reduction method is introduced, which can reduce the dimensionality of high-dimensional space points while maintaining the original low-dimensional structure. For example, a highdimensional eigenvector $\tilde{D}_{i}$ with $j$ dimensions projects the data onto a hyperplane, and introduce the kernel function

$$
\kappa\left(x_{i}, x_{j}\right)=\phi\left(x_{i}\right)^{\mathrm{T}} \phi\left(x_{j}\right)
$$

After that, use the following formula to derive the low-dimensional eigenvector $\tilde{D}_{i}{ }_{i}$ after dimensionality reduction:

$$
\tilde{D}_{i}^{\prime}=\tilde{D}_{i}^{\mathrm{T}} \phi(x)
$$

Stage 5: Evaluation of teachers' teaching ability: The evaluation index $T$ can be calculated by the following formula:

$$
T=W^{\mathrm{T}} V
$$

where, $T$ represents the final evaluation value of a teacher's teaching ability, $W$ the weight vector, and $V$ the teacher's teaching ability index vector. 


\subsection{Applicability analysis of hidden Markov model to the evaluation of PE students' learning effect in colleges}

Hidden Markov model in the field of machine learning, a dynamic Bayesian network with simple structure, has been widely used in time series data modeling and widely recognized especially in the fields of image processing, speech recognition and natural language processing. The hidden Markov model can be used to estimate and infer the difficult and unobservable variables based on the observed variables, thus it is especially suitable for measuring the mastery of students' knowledge of a series of knowledge points in the teaching process of PE courses in colleges.

Due to the discrepancy in students' abilities and the large differences in the forms of professional PE projects, traditional testing methods often fail to evaluate students' actual learning effects in real time and with high accuracy. The hidden Markov model in the field of machine learning can just use the probabilistic graphical model to estimate the unknown variables using observable known variables. Among them, the observable known variables are called observation variables, and the unknown variables are called hidden variables. Figure 1 shows the graph structure of the hidden Markov model.

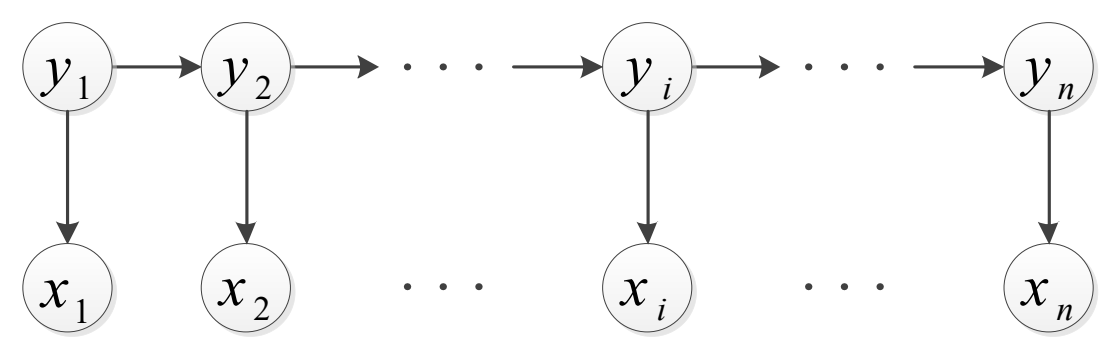

Fig. 1. Graph structure of the hidden Markov model

In Figure 1 , the hidden variable $\left\{y_{1}, y_{2}, \cdots, y_{n}\right\}$ represents the degree of mastery of a series of knowledge points, and the observation variable $\left\{y_{1}, y_{2}, \cdots, y_{n}\right\}$ represents the test results for a series of knowledge points. In general, the hidden Markov model calculations suitable for evaluating students' learning effects can be divided into the following four stages.

Stage 1: Define the joint probability distribution: The joint probability distribution of the hidden Markov model shown in Figure 1 can be defined as:

$$
P\left(x_{1}, y_{1}, \cdots, x_{n}, y_{n}\right)=P\left(y_{1}\right) P\left(x_{1} / y_{1}\right) \prod_{i=2}^{n} P\left(y_{i} / y_{i-1}\right) P\left(x_{i} / y_{i}\right)
$$

Stage 2: Define the state transition probability, output observation probability, and initial state probability: State transition probability refers to the model's probability of state transition between two knowledge points before and after itself, output observation probability refers to the probability of test score of each knowledge point in the current state, and the initial state probability refers to the 
probability of each knowledge point test score in the initial state. The three probabilities are shown as in formulas (7-9):

$$
\begin{gathered}
a_{i j}=P\left(y_{2}=j / y_{1}=i\right) \\
b_{i j}=P\left(y_{1}=j / y_{1}=i\right) \\
c_{i}=P\left(y_{1}=i\right)
\end{gathered}
$$

Stage 3: Iterate: First, select the initial state according to the initial state probability $c_{i}$; then, select an observation variable according to the initial state and the output observation probability $b_{i j}$; finally, determine the next variable state based on the initial state and the state transition probability $a_{i j}$.

Stage 4: Hidden variables: When all the knowledge points are iterated, the result $\left\{y_{1}, y_{2}, \cdots, y_{n}\right\}$ is the state of the hidden variables.

\section{PE Teaching Evaluation Model Based on Hybrid Technology}

\subsection{Flow chart of the evaluation model}

Based on hybrid technology of data mining and hidden Markov model, this study presents a new evaluation model for PE teaching in colleges, of which the flow chart is shown in Figure 2.

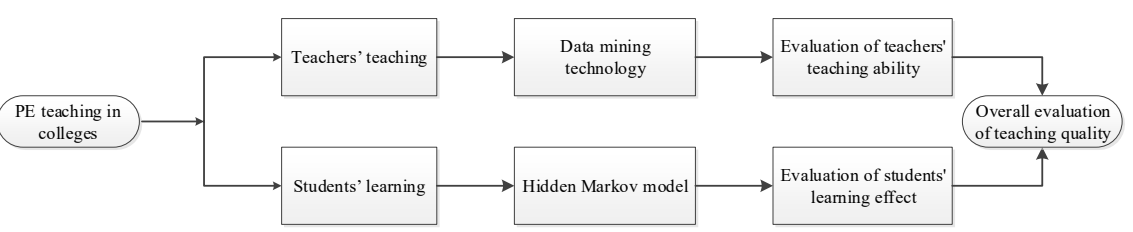

Fig. 2. Flow chart of the evaluation model for PE teaching in colleges based on the hybrid technology of data mining and hidden Markov model

It can be seen from Figure 2 that the PE teaching evaluation model proposed in this study includes two aspects: teachers' teaching and students' learning. As to the former, evaluation of teachers is completed through the data mining technology. As to the latter, the evaluation of students is completed through the hidden Markov model. Finally, the overall evaluation of teaching quality is obtained by integrating the evaluation of teachers' teaching ability and the evaluation of students' learning effect.

\subsection{Preparation of data before using hybrid technology}

The data preparation includes two parts: The data preparation before evaluating teachers' teaching ability, and that before evaluating students' learning effect. 
First, let's introduce how to prepare data for evaluating teachers' teaching ability. The preparation of evaluation data of teachers' teaching ability can be divided into the following three steps.

- Step 1: Define the layers of teaching ability evaluation scale of PE teachers in colleges. The layers of the scale can be divided into three types: single-layer, double-layer or multiple-layer. This study chooses to use a single-layer evaluation scale.

- Step 2: Define the meaning of each index in the evaluation scale. There are 10 indexes used in this study, such as teachers' highest degree, teacher's professional title, business test results and scientific research achievements. The names and interpretations of specific index parameters are shown in Table 1.

Table 1. Various indexes in the teaching ability evaluation scale of professional PE teachers for postgraduates in colleges

\begin{tabular}{|c|l|}
\hline Name & \\
\hline $\mathrm{I}_{1}$ & Highest degree \\
\hline $\mathrm{I}_{2}$ & Professional title \\
\hline $\mathrm{I}_{3}$ & Business test results \\
\hline $\mathrm{I}_{4}$ & Scientific research achievements \\
\hline $\mathrm{I}_{5}$ & Employment time \\
\hline $\mathrm{I}_{6}$ & Carefulness in lesson preparation \\
\hline $\mathrm{I}_{7}$ & Classroom interaction \\
\hline $\mathrm{I}_{8}$ & Consistency of knowledge points \\
\hline $\mathrm{I}_{9}$ & Business awards received \\
\hline $\mathrm{I}_{10}$ & Satisfaction with after class Q \& A \\
\hline
\end{tabular}

Step 3: Quantify teachers' data. The teachers' data used either for training or testing are quantified according to the indexes shown in Table 1 so as to facilitate the training and testing of the machine learning model.

Then, let's introduce how to prepare data for evaluating students' learning effect. The preparation of the student's learning evaluation data is specifically as follows in steps 4 to 6 .

Step 4: Determine course knowledge points. Based on the requirements of the syllabus and the experience of experts in the field, the knowledge points involved in a PE course are listed in detail.

Step 5: Determine the logical relationship between knowledge points. According to actual classroom teaching practice and the order in which the students accept the knowledge points, the logical relationship between all the knowledge points is determined. For example, Figure 3 shows the logical relationship between four knowledge points.

In Figure 3, the knowledge point 1 is the preceding knowledge of the knowledge point 2, which in turn is the preceding knowledge of the knowledge points 3 and 4.

Step 6: Record the students' written test scores for the mastery of PE knowledge in the non-competitive part. Students' mastery of knowledge points can only be presented through questions in class or written tests. That is to say, students' mastery of 
knowledge points is a hidden variable that is difficult to observe. These hidden variables can only be expressed through the observable variables such as questions in class or written test scores. Because the classroom Q\&A records are not easy to standardize, this study uses written test scores as observation variables.

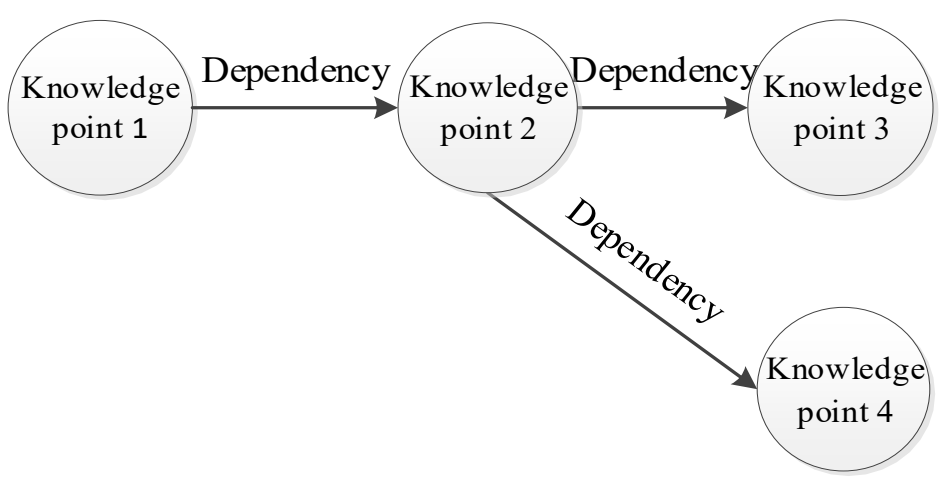

Fig. 3. Logical relationship between four knowledge points

\subsection{Parallel processing in the use of hybrid technology}

The parallel processing calculation also includes two parts: one is to evaluate the teachers' teaching ability, and the other to evaluate the students' learning effect.

First, let's introduce the processing calculation for evaluating teachers' teaching ability. This process can be divided into the following 2 steps.

Step 1: Calculate the weights of the indexes listed in Table 1. Collect the real data of a certain amount of teachers as training data. After the quantitative processing operation, use the data mining technology introduced in Section 1.1 of this study to obtain the corresponding weights $\mathrm{W} 1 \sim \mathrm{W} 2$ of each indicator I1 I10 listed in Table through training.

Step 2: Calculate the ability of the teachers to be evaluated. As for the data of the teachers to be evaluated, first complete the preparation of the data according to Section 2.2, and then perform weighted accumulation of each indicator data. The results are the scores of teaching ability of the teachers to be evaluated. That is, the score $S_{T}$ of a teacher's teaching ability can be calculated by the following formula:

$$
S_{T}=\sum_{i=1}^{10} I_{i} \times W_{i}
$$

Then, let's introduce the processing calculation for evaluating the students' learning effect. The processing calculation of this part is specifically shown by the following steps 3 to 5 .

Step 3: Stipulate and simplify knowledge dependencies. Based on the data preparation in Section 2.2, the knowledge dependency will be further processed. Due to the 
intricate relationship between knowledge points, proper stipulation and simplification of the logical relationship between knowledge points without affecting the generality will help to better apply the hidden Markov model. Taking Figure 3 as an example, the logical relationship between the four knowledge points listed in the figure are stipulated and simplified, and a new logical relationship is formed as shown in Figure 4. In Figure 4, the preceding knowledge of the knowledge point 4 is changed from the knowledge point 2 to the knowledge point 3 . After the stipulation, although the knowledge dependency graph is slightly changed, the overall evaluation effect is better because the knowledge framework can be applied to the hidden Markov model as a whole.

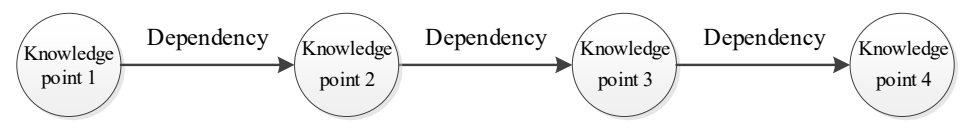

Fig. 4. Logical relationship between knowledge points after stipulation and simplification

Step 4: Construct a hidden Markov model between knowledge points and tests. Assuming that the knowledge points 1 to 4 shown in Figure 4 correspond to the tests 1 to 4, a hidden Markov model composed of the knowledge points and the tests is as shown in Figure 5.

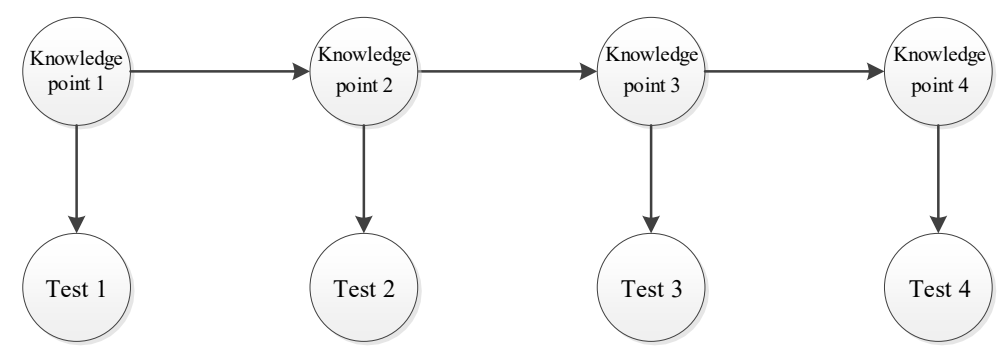

Fig. 5. Hidden Markov model between knowledge points and tests

Step 5: Calculate the learning effect of the students to be evaluated. For the hidden Markov model that has been constructed, the Hidden Markov Model hidden variable calculation method introduced in Section 1.1 of this study is used to evaluate the students' learning effect. The evaluation score SS is as follows:

$$
S_{S}=\sum_{i=1}^{n} K_{i} \times V_{i}
$$

where, $n$ indicates the number of knowledge points, $K_{i}$ the learning effect evaluation score of the $i$-th knowledge point, and $V_{i}$ the weight of the learning effect evaluation of the $i$-th knowledge point. 


\subsection{Generation of overall evaluation after using hybrid technology}

After obtaining the evaluation score of teachers' teaching ability, $S_{T}$, and that of students' learning effect, $S_{T}$, the overall score of PE teaching evaluation can be given by the following formula:

$$
S=S_{T} \times 0.7+S_{S} \times 0.3
$$

where, the weight of $S_{T}$ is set to 0.7 , and that of $S_{S}$ is set to 0.3 . That is, this study holds that students are the main body of teaching activities, compared with the evaluation of teachers' teaching ability, the evaluation of students' learning effect is more important.

\section{$4 \quad$ Experimental Results and Analysis}

The experimental process hardware configuration of the above-mentioned hybrid technology-based PE teaching evaluation model is as follows: CPU with i3-5005U 2.0 GHz, and 4G-RAM. The experimental software selects Matlab 2013b, and the software running system selects Windows 10 flagship edition. With the PE basic course in a college selected as research object, this experiment collects the teaching and learning information of $15 \mathrm{PE}$ teachers and 462 students of different majors in 15 classes participating in the teaching process. According to the method given in Section 2 of this paper, the overall evaluation of PE teaching is obtained by taking individual students as a unit. Then, carry out the survey on the acceptance of the overall teaching evaluation acceptance of the teacher and students through questionnaires. When the acceptance error is within $\pm 5 \%$, the calculated overall evaluation is considered to be accurate.

In order to verify the accuracy of the algorithm in this study, it is compared with the algorithm choices applied in other subject areas: the algorithms [11-14]. The implementation process of the algorithm is shown in the original literature. The comparison results are shown in Figure 6.

According to the comparison of the accuracy of several algorithms shown in Figure 6 , the algorithm in this study has the highest accuracy $(90.3 \%)$, which is obviously better than the other algorithms selected. The algorithm proposed with Siegel \& Dubé [13] has the lowest accuracy (only 68.3\%), because the algorithm is only designed for $\mathrm{PE}$ in the education industry, and its application is narrow. The accuracy of the algorithms proposed with Campbell [11], Bernhardt [12] (75.9\% and70.7\%, respectively) is slightly higher than Becerra-Fernández et al. [6], which is due to the wider industrial application of the algorithms proposed with Campbell [11], Bernhardt [12]. Moreover, the algorithm proposed with Hirsch et al. [3] is more suitable for the teaching of basic theories and methods of PE teaching, and the algorithm proposed with Bernhardt [12] is more suitable for PE teaching in exercising, training and competition skills. The accuracy of the algorithm proposed with Karakus \& Türkkan [14] is second only to the algorithm proposed in this study $(81.0 \%)$, which is because the algo- 
rithm optimizes the design of the evaluation scale and can reflect the real teaching effect well.

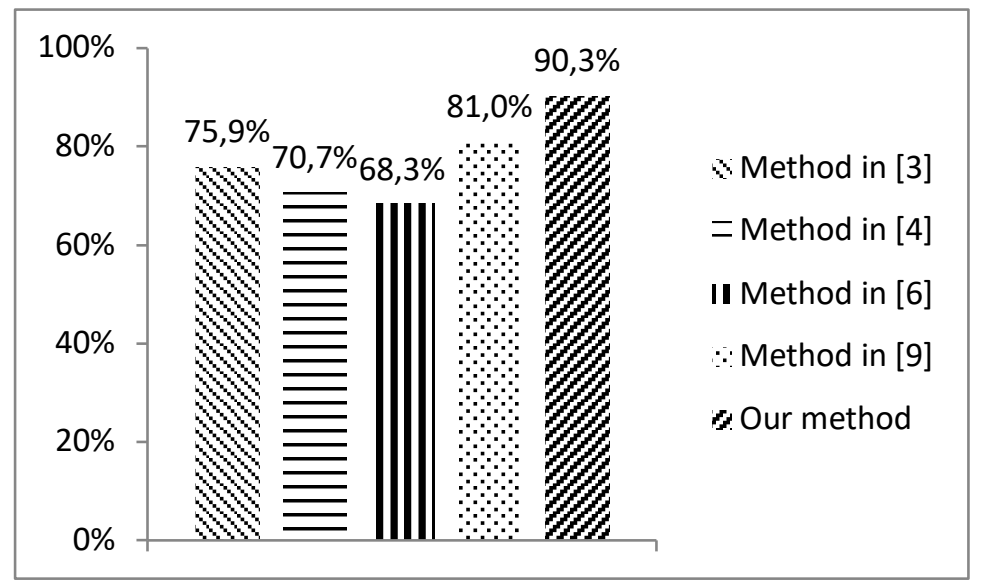

Fig. 6. Comparison of the accuracy of previous algorithms and the algorithm in this study

In addition, the running time of the selected algorithm is compared with the computational complexity of each algorithm, and the experimental results are shown in Figure 7.

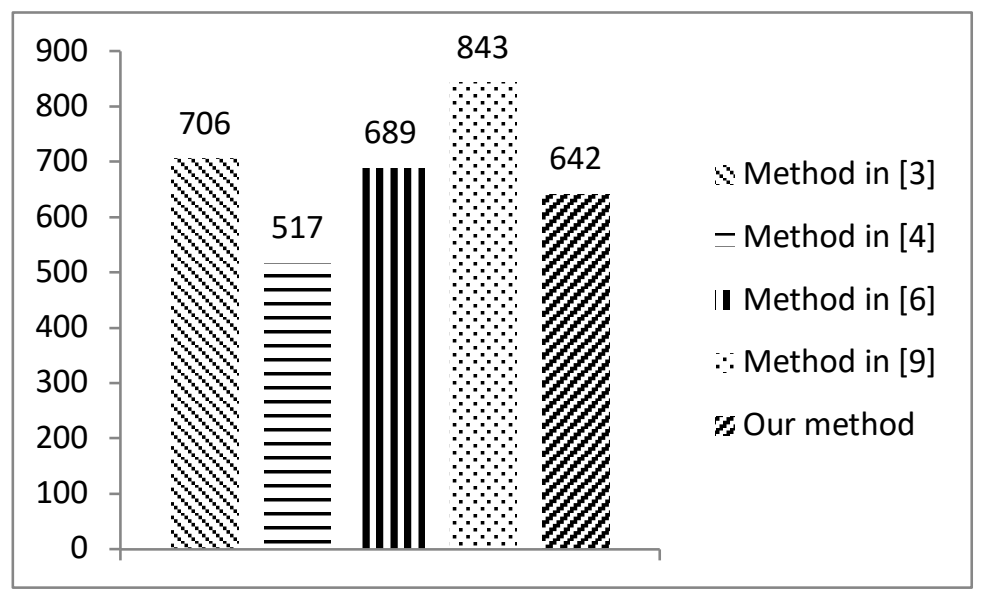

Fig. 7. Comparison of running time between previous algorithms and the one used in this study (Unit: second)

According to Figure 7 that shows the experimental comparison of running time between these algorithms, although the speed of the selected algorithm (642 seconds) is not as good as the algorithm proposed with Bernhardt [12] (517 seconds), it has a better performance among all algorithms. Particularly, its running time is significantly less than the algorithms proposed with Campbell [11] (706 seconds) and Karakus \& 
Türkkan [14] (843 seconds). The algorithm proposed with Karakus \& Türkkan [14] has the longest running time, because the algorithm needs to process a large number of high redundancy tables, which increases the number of memory accesses geometrically and results in the lower efficiency of the algorithm. The algorithms proposed with Campbell [11] and Siegel \& Dubé [13] are of moderate operation efficiency, because the algorithms proposed with Campbell [11] and Siegel \& Dubé [13] are designed for specific learning contents, and a part of their operation efficiency is sacrificed for ensuring accuracy. It should be particularly pointed out that the running time of the algorithm proposed with Bernhardt [12] is less than that of the algorithm proposed in this study, because the former requires fewer tables and is optimized in terms of efficiency.

In summary, the algorithm proposed in this study has achieved better overall performance than the previous algorithms in terms of accuracy and rapidity.

\section{Conclusion}

This study proposes a research method for PE teaching evaluation based on hybrid technology of data mining and hidden Markov model, obtains the evaluation of teachers' teaching ability using data mining technology in the field of machine learning and the evaluation of students' learning effect using the hidden Markov model in the field of machine learning, and finally gets the overall evaluation of PE teaching in colleges through the weighted sum of the evaluations in these two aspects. This study is innovative in the following two aspects. On the one hand, it carries out the quantitative analysis and calculation of the teaching process with the introduction of more mature technologies in the field of machine learning. On the other hand, it respects the teaching practice by taking students as the main body, and carries out teaching evaluation in terms of both teachers and students. The experimental results show that the proposed algorithm has better accuracy and higher computational efficiency in PE teaching evaluation. The next step is to optimize the algorithm flow appropriately in order to further improve the computational efficiency of the algorithm.

\section{References}

[1] Kilbom, A. (1971). Physical training in women. Scandinavian Journal of Clinical and Laboratory Investigation, Supplement 119, 1-34.

[2] Saltin, B. (1971). Guidelines for physical training. Scandinavian Journal of Rehabilitation Medicine, 3(1), 39-46.

[3] Hirsch, S.E., Healy, S., Judge, J.P., Lloyd, J.W. (2016). Effects of an interdependent group contingency on engagement in physical education. Journal of Applied Behavior Analysis, 49(4), 975-979. https://doi.org/10.1002/jaba.328

[4] Erbaş, M.K., Ünlü, H. (2016). The relationship between the levels of physical education predisposition and motor skills of adolescent female students. Turkish Online Journal of Educational Technology, Special Issue, 1091-1098.

[5] Gråstén, A. (2016). Children's expectancy beliefs and subjective task values through two years of school-based program and associated links to physical education enjoyment and 
physical activity. Journal of Sport and Health Science, 5(4), 500-508. https://doi.org/10. 1016/j.jshs.2015.12.005

[6] Becerra-Fernández, C.A., Merino-Marban, R., Mayorga-Vega, D. (2016). Effect of a physical education-based dynamic stretching program on hamstring extensibility in female high-school students. Kinesiology, 48(2), 258-266.https://doi.org/10.26582/ k.48.2.3

[7] Abdullah, M.Y., Hussin, S., Shakir, M. (2018). The effect of peers' and teacher's Efeedback on writing anxiety level through CMC applications, International Journal of Emerging Technologies in Learning, 13(11), 196-207. https://doi.org/10.3991/ ijet.v13i11.8448

[8] Lohbeck, A., Engels, E.S., Freund, P.A. (2019). Assessing students' enjoyment in physical education: measurement invariance across school tracks and relationships with grades. Journal of Psychoeducational Assessment, 37(8), 1023-1029. https://doi.org/10. $\underline{1177 / 0734282918804600}$

[9] Gubby, L. (2019). Can korfball facilitate mixed-PE in the UK? The perspectives of junior korfball players. Sport, Education and Society, 24(9), 994-1005. https://doi.org/10. $\underline{1080 / 13573322.2018 .1519506}$

[10] Bin, H. (2017). Factor analysis of physical quality evaluation and physical education curriculum model optimization for college students based on questionnaire investigation. Boletin Tecnico/Technical Bulletin, 55(20), 365-372.

[11] Campbell, R.N. (1970). An evaluation and comparison of present methods for teaching English grammar to speakers of other languages. Tesol Quarterly, 4(1), 3748.https://doi.org/10.2307/3585777

[12] Bernhardt, S.A. (1992). Teaching English: Portfolio evaluation. Clearing House, 65(6), 333-334. https://doi.org/10.1080/00098655.1992.10114238

[13] Siegel, J., Dubé, L.S. (1982). An evaluation of integrated service English teaching. Elt Journal, 36(3), 152-163. https://doi.org/10.1093/elt/36.3.152

[14] Karakus, M., Türkkan, B.T. (2017). Investigating the needs for measurement and evaluation course: a case study on English language teaching program. Journal of Education \& Training Studies, 5(4), 227-239. https://doi.org/10.11114/jets.v5i4.2205

\section{$7 \quad$ Author}

Yuansheng Zeng, male, is working as Institute of Physical Education, Chongqing Technology and Business University, Chongqing 400067, China, with the research interest in physical education. Email: 895381769@qqq.com

Article submitted 2019-10-27. Resubmitted 2019-11-28. Final acceptance 2019-11-28. Final version published as submitted by the authors. 